\title{
Importancia de las enfermedades infecciosas para la conservación de la fauna silvestre amenazada de Chile
}

\section{Importance of infectious disease for the conservation of Chilean threatened wildlife}

\author{
Andrés Valenzuela-SÁnchez ${ }^{1 *} \&$ Gonzalo Medina-Vogel ${ }^{1}$ \\ ${ }^{1}$ Facultad de Ecología y Recursos Naturales, Universidad Andres Bello, República \#440, Santiago, Chile. \\ *E-mail: a.valenzuela.s@uandresbello.edu \\ Título abreviado: Enfermedades infecciosas en fauna silvestre.
}

\begin{abstract}
RESUMEN
En conservación biológica existe un incremento en los antecedentes de que las enfermedades infecciosas (EIs) pueden afectar especies silvestres causando mortalidades que pueden aumentar el riesgo de extinción. Es así que para conseguir metas en conservación animal hoy se estima necesario conocer el rol de los patógenos en las poblaciones silvestres. En concordancia con lo anterior, los objetivos de este trabajo fueron: 1) determinar la contribución de las EIs a la declinación de las especies amenazadas de la fauna silvestre presente en Chile, de acuerdo a lo señalado por la Lista Roja de la UICN, 2) evaluar la evidencia existente en la literatura científica del impacto de las EIs en estas especies, y 3) proponer el nivel de riesgo para cada especie animal amenazada de Chile de ser impactada por EIs que podrían provocar potenciales declinaciones poblacionales o extinción. En Chile, 105 especies animales se encuentran en alguna categoría de amenaza. En el 16,2 \% $(N=17)$ de estas especies se menciona, según la Lista Roja de la UICN, alguna EI como principal amenaza para su conservación. Pese a esto, no existe evidencia en la literatura científica indexada (ISI-wok) de alguna EI provocando declinaciones poblacionales en el país. Se sugiere que los anuros Telmatobius zapahuirensis y T. pefauri, y el cánido Lycalopex fulvipes requieren esfuerzos urgentes de investigación epidemiológica debido a su categoría de conservación y distribución restringida, lo que podría aumentar el riesgo de extinción mediado por EIs. Finalmente, se plantean direcciones futuras en el estudio de las EIs que contribuyan a alcanzar la meta de la conservación biológica en Chile.
\end{abstract}

Palabras Clave: Conservación, patógeno, declinación poblacional, extinción, UICN.

\begin{abstract}
There is evidence that infectious diseases (IDs) can directly affect wildlife even causing catastrophic died-offs, which increase extinction risk. In this way, to achieve the biological conservation goal we should know the role of IDs on wild populations. The aim of this work was 1) to determine the contribution of IDs in Chilean wildlife declination, according to the IUCN Red List, 2) to evaluate the level of scientific evidence of this threat on wildlife from Chile, and 3) propose a risk level (for each threatened wildlife species) of become threatened by an ID with potential to leading population decline or extinction. In the country, 105 species are in some category of threat, of which $16.2 \%(\mathrm{~N}=17)$ have IDs as potential major threat. Despite this, no evidence is available into scientific literature about an ID producing population declines in Chile. We suggest that anuran species Telmatobius zapahuirensis and T. pefauri, and the canid Lycalopex fulvipes, need urgent efforts in epidemiological research; due in part by its restricted distribution and conservation status, which could increase risk of extinction driven by IDs. By the end, we suggest future directions to enhance the biological conservation in Chile.
\end{abstract}

KeYwords: Conservation, extinction, IUCN, pathogens, population decline.

\section{INTRODUCCIÓN}

Actualmente la biodiversidad en el planeta Tierra enfrenta una crisis global, caracterizada por declinaciones poblacionales alarmantes y una tasa de extinción en animales 100 a 1000 veces mayor que la tasa histórica (May 2010, Millenium Ecosystem Assessment 2005). Los conductores de extinción en animales son diversos, dentro de los cuales se pueden citar la pérdida y fragmentación del hábitat, la introducción de especies alóctonas, la sobreexplotación, la contaminación, el cambio climático y las enfermedades infecciosas (Millenium Ecosystem Assessment 2005).

Pese a lo anterior, el rol de las enfermedades infecciosas (EIs) 
en la conservación de la fauna silvestre fue "radicalmente subestimado" (sensu Leopold 1933) por los primeros conservacionistas (Gaydos \& Gilardi 2004). Es así como la evidencia histórica de causalidad de extinción mediada por EIs es mayormente anecdótica y a menos del $4 \%$ de las especies de plantas y animales enlistadas como extintas desde 1500 se les ha mencionado alguna(s) EI(s) como factor contribuyente de su extinción (Smith et al. 2006). Sin embargo, la evidencia de declinaciones poblacionales y extinción mediada por patógenos ha aumentado considerablemente en los últimos años, por lo que se espera que la incorporación de las EIs entre los factores de amenaza para la conservación de la biodiversidad aumente significativamente en el futuro (Medina-Vogel 2010; Smith et al. 2009; Daszak et al. 2001, 2003; Lafferty 2003). Por ejemplo, en 1993-1994, una epidemia de distemper canino en leones (Phantera leo Linnaeus 1758) exterminó al $30 \%$ de una población que era intensamente monitoreada en el Parque Nacional Serengeti (Cleaveland et al. 2000). Así mismo, el 2005 emergió en Reino Unido una nueva enfermedad fatal en los pinzones, causada por el protozoo Trichomonas gallinae Rivolta 1878. Para el 2007, en la zona geográfica con más alta incidencia, la enfermedad había disminuido entre el $35 \%$ y el $21 \%$ las poblaciones reproductivas de Carduelis chloris Linnaeus 1758 y Fringilla coelebs Linnaeus 1758, respectivamente, representando una mortalidad que excedió el medio millón de aves (Robinson et al. 2010). En África, en la región fronteriza de Gabón y República del Congo, entre el 2001 y 2003, el virus Ébola produjo una declinación del $80 \%$ de las poblaciones de chimpancé y gorila, y es considerado actualmente una seria amenaza para las poblaciones remanentes de grandes simios que habitan los bosques lluviosos del centro del continente (Leendertz et al. 2006).

En ciertas ocasiones las EIs pueden producir la extinción completa de una especie (Ver Smith et al. 2006), especialmente en aquellas que se encuentran previamente afectadas por amenazas concomitantes (Smith et al. 2006, 2009; Pedersen et al. 2007; Lafferty 2003). Por ejemplo, los últimos individuos del gastrópodo Partula turgida Pease 1864, que eran criados en cautividad debido a la desaparición de las poblaciones silvestres a consecuencia de la introducción del caracol carnívoro Euglandina rosea Férussac, 1821 en la Polinesia Francesa, murieron por causa de la infección con el microsporidio Steinhausia sp. (Cunningham \& Daszak 1998).

Ejemplos como los citados anteriormente resaltan la importancia que reviste para la conservación animal el conocimiento de los efectos que representan las EIs en las poblaciones silvestres (Deem et al. 2001). En este sentido, Smith et al. (2009) proponen una aproximación que combine evidencia y teoría para estimar el efecto relativo y la probabilidad de amenaza de las EIs en las especies animales, mediante el a) reconocimiento de las especies hospederos que están en mayor riesgo de extinción mediada por enfermedad, b) el patógeno más probable de causar esta enfermedad, y c) su interacción con otros conductores de extinción. En concordancia con lo anterior, los objetivos de este trabajo son 1) determinar la contribución de las EIs a la declinación de las especies amenazadas de la fauna silvestre presente en Chile, de acuerdo a lo señalado por la Lista Roja de la UICN, 2) evaluar la evidencia existente en la literatura científica del impacto de las EIs en estas especies, y 3) proponer el nivel de riesgo para cada especie animal amenazada de Chile de ser impactada por EIs que puedan provocar potenciales declinaciones poblacionales o extinción.

\section{MATERIALES Y MÉTODOS}

En septiembre de 2012 se realizó una búsqueda de todas las especies animales presentes en Chile enlistadas en una categoría de amenaza (Extinto, Extinto en la Naturaleza, En Peligro Crítico, En Peligro y Vulnerable) en la Lista Roja de la UICN (www.iucnredlist.org). La lista roja destaca especies que están en gran riesgo de extinción, clasificándolas dentro de una categoría de amenaza. Esta clasificación es basada en datos y criterios objetivos, donde expertos recopilan y revisan la información primaria requerida para incluir a cada especie dentro de una categoría; utilizando datos publicados y no publicados, y generalmente aportes de uno o más asesores. La utilidad de la lista roja no solo deriva de la clasificación de cada especie dentro de una categoría de amenaza, sino que además de la gran cantidad de información colectada para elaborar estas evaluaciones (Rodrigues et al. 2006). Por este motivo, con la finalidad de determinar cuáles de las especies animales presentes en Chile poseían como amenaza las EIs, se realizó una búsqueda individual de la información detallada en "Major Threat(s)". A pesar que las amenazas pueden ser citadas como ocurriendo en el pasado, presente o con posibilidades de ocurrir en el futuro (Smith et al. 2006), no realizamos distinción en la temporalidad de la amenaza al momento de clasificar una especie como amenazada por EIs. Para evaluar si existían diferencias en el porcentaje de especies amenazadas por EIs entre las clases taxonómicas, se utilizó la Prueba exacta de Fisher. Posteriormente, con las especies que se encontraban amenazadas por EIs se llevó a cabo una búsqueda bibliográfica individual en la plataforma Thomson Reuters ISI-Web of Knowledge (ISI-wok, artículos entre 1988-2014) para así evaluar la evidencia presente en la literatura científica sobre el impacto de las EIs en estas especies. Nosotros utilizamos el término "patógeno" para incluir tanto macro como micro parásitos, y el término "enfermedad infecciosa" para representar una enfermedad 
causada por un "patógeno" contagioso (Pedersen et al. 2007).

Finalmente, con el objetivo de proponer el riesgo de enfrentar declinaciones poblacionales o extinción mediada por EIs, se realizó una búsqueda en el ISI-wok (artículos entre 1988-2012) modificada de Smith et al. (2006) incluyendo Clase, Orden, Familia y Genero de cada especie amenazada y los siguientes tópicos: (nivel taxonómico respectivo*) AND (declin* OR extinct* OR threat* OR endanger* OR mass mortality*) AND (infectious disease* OR pathogen* OR bacteri* OR parasit* OR fung* OR protozoa*) NOT (human). Los resúmenes o los manuscritos completos fueron obtenidos desde los artículos que contenían información describiendo mortalidad en masa, declinación poblacional o extinción mediada parcial o totalmente por EIs; situación que fue calificada como presencia de evidencia. Además, si algún trabajo citado en estos artículos hacía mención a los tópicos de nuestra búsqueda, estos trabajos también fueron incluidos en la base de datos. Con la información generada desde la búsqueda bibliográfica, y basados en una aproximación de relación taxonómica, se le asignó a cada especie amenazada de Chile un nivel de riesgo de la siguiente categoría: Muy Alto (evidencia a nivel de género), Alto (evidencia a nivel de familia), Medio (evidencia a nivel de orden), Bajo (evidencia a nivel de clase) y Muy bajo (sin evidencia en ningún nivel taxonómico). Para la determinación del "Nivel de riesgo de amenaza por EIs" y debido a que el objetivo del trabajo se limitó a poblaciones silvestres amenazadas, excluimos de nuestra base de datos toda evidencia proveniente de animales mantenidos en cautividad.

\section{RESULTADOS}

ESPECIES ANIMALES AMENAZADAS EN CHILE: CONTRIBUCiÓN DE LAS ENFERMEDADES INFECCIOSAS

Según la Lista Roja de la UICN, ninguna especie animal silvestre presente en Chile ha sido declarada como Extinta o Extinta en la Naturaleza. Por otra parte, existen 105 especies animales en alguna categoría de amenaza (Fig. 1). En el 16,2\% $(N=17)$ de estas especies se menciona alguna EI como principal amenaza para su conservación (Fig. 1, Tabla 1). La distribución de las especies amenazadas por EIs entre clases taxonómicas no es homogénea (Prueba exacta de Fisher $P<0,001$ ), porcentualmente y en relación con su clase los antozoos poseen más especies amenazadas por EIs, seguidos consecutivamente por los anfibios, mamíferos y aves.

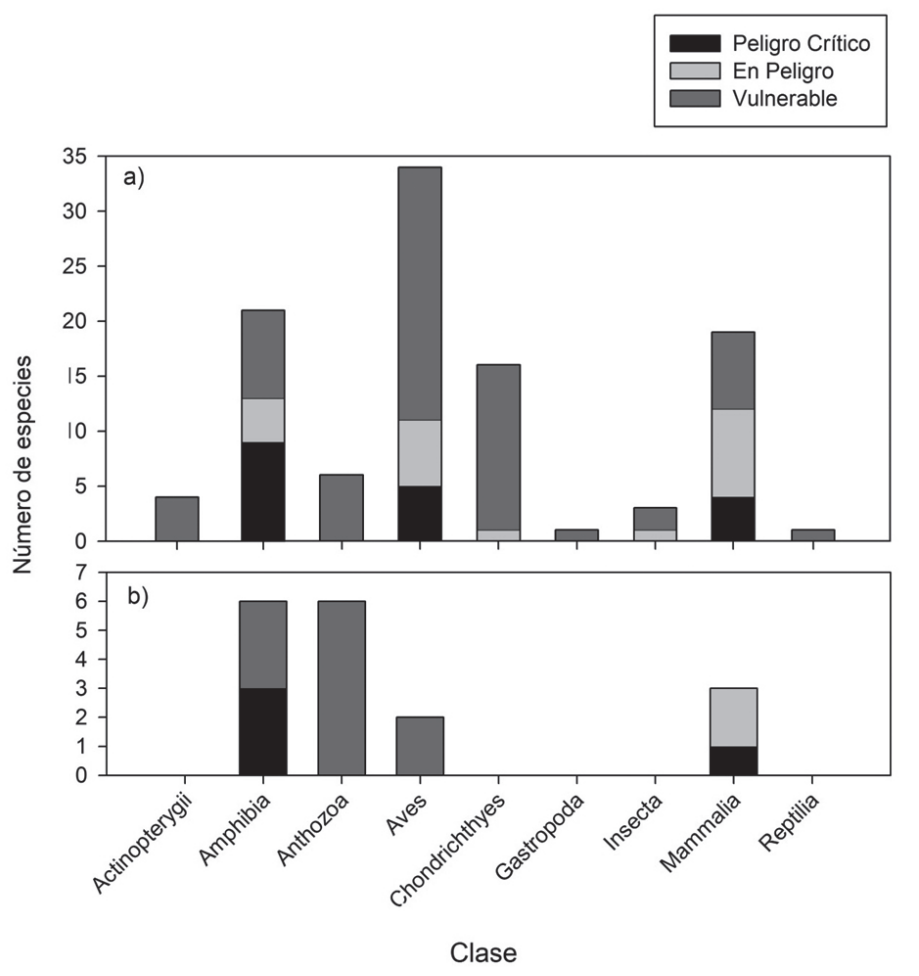

Figura 1. Número de especies animales de Chile (por clase taxonómica) a) en alguna categoría de amenaza y b) para las cuales las EIs fueron señaladas como principal amenaza para su conservación. Los datos fueron obtenidos desde el sitio web de la Lista Roja de la UICN.

FIGURE 1. Number of Chilean wildlife species (by taxonomic class) a) in a threat category and b) for which infectious diseases are identified as a major threat for its conservation. Data was obtained from the IUCN Red List website. 
Un detalle de las especies amenazadas, así como de la etiología propuesta en la lista roja se muestra en la Tabla 1.

Evidencia EN LA Literatura CiENTífica PARA CHILE

Clase Anthozoa: La búsqueda por especie en ISI-wok no arrojó resultados. Cabe destacar que las especies de antozoos amenazados en Chile, para los cuales una EI fue sindicada como principal amenaza, poseen una distribución que en el país se restringe solamente a la Isla de Pascua.

Clase Amphibia: Bourke et al. (2010) describen la presencia de Batrachochytrium dendrobatidis Longcore, Pessier \& Nichiols, 1999 ( $B d$, agente etiológico de la quitridiomicosis) en individuos de Rhinoderma darwinii Duméril \& Bibron

TABla 1. Especies de fauna silvestre de Chile en las que se menciona a las enfermedades infecciosas como principal amenaza para su conservación, según la Lista Roja de la UICN.

TABLE 1. Chilean wildlife species for which infectious diseases are identified as a major threat for its conservation (IUCN Red List).

\begin{tabular}{|c|c|c|c|c|c|}
\hline Clase & ESPECIE & $\begin{array}{c}\text { Estado DE } \\
\text { CONSERVACIÓN }\end{array}$ & ENDEMISMO & DistriBUCIÓN & ENFERMEDAD PROPUESTA UICN \\
\hline \multirow{6}{*}{$\frac{. \frac{\pi}{0}}{\frac{0}{\frac{Z}{2}}}$} & Rhinoderma rufum & $\mathrm{CE}$ & $\mathrm{Si}$ & Chile & Quitridiomicosis \\
\hline & R. darwinii & VU & No & Chile, Argentina & Quitridiomicosis \\
\hline & Telmatobius marmoratus & VU & No & $\begin{array}{c}\text { Chile, Perú, } \\
\text { Bolivia }\end{array}$ & Quitridiomicosis \\
\hline & T. pefauri & $\mathrm{CE}$ & $\mathrm{Si}$ & Chile & Quitridiomicosis \\
\hline & T. peruvianus & VU & No & Chile, Perú & Quitridiomicosis \\
\hline & T. zapahuirensis & $\mathrm{CE}$ & $\mathrm{Si}$ & Chile & Quitridiomicosis \\
\hline \multirow{6}{*}{ 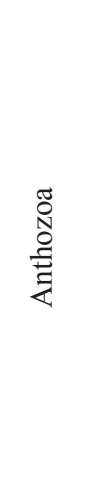 } & Acropora listeri & VU & No & Global & Blanqueamiento \\
\hline & Montipora australiensis & VU & No & Hemisferio sur & Enfermedad del coral \\
\hline & M. caliculata & VU & No & Hemisferio sur & Enfermedad del coral \\
\hline & M. crassituberculata & VU & No & Hemisferio sur & Enfermedad del coral \\
\hline & Pocillopora danae & VU & No & Hemisferio sur & Enfermedad del coral \\
\hline & Psammocora stellata & VU & No & Global & Enfermedad del coral \\
\hline \multirow{2}{*}{$\sum^{\infty}$} & Eudyptes chrysocome & VU & No & Hemisferio sur & $\begin{array}{c}\text { Cólera aviar, intoxicación por } \\
\text { FANs }\end{array}$ \\
\hline & E. chrysolophus & VU & No & Hemisferio sur & No determinada \\
\hline \multirow{3}{*}{ 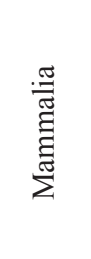 } & Lycalopex fulvipes & $\mathrm{CE}$ & $\mathrm{Si}$ & Chile & No determinada \\
\hline & Hippocamelus bisulcus & $\mathrm{E}$ & No & Chile, Argentina & $\begin{array}{c}\text { Brucelosis, Fiebre aftosa, } \\
\text { Cisticercosis }\end{array}$ \\
\hline & Balaenoptera borealis & $\mathrm{E}$ & No & Global & Deterioro y pérdida de barbas \\
\hline
\end{tabular}


1841 de vida silvestre y mantenidos en cautividad. Además, existe evidencia de que la quitridiomicosis pudo haber causado un evento de $100 \%$ de mortalidad en un grupo de 30 individuos de $R$. darwinii exportados a Alemania en 2007, sin embargo los efectos de factores estresantes en la mortalidad de los individuos, como el viaje y la mantención en cautividad, no pudieron ser separados (Bourke et al. 2010, Werning 2009). Soto-Azat et al. (2013a) realizaron un estudio retrospectivo de infecciones con $B d$ en más de 600 anfibios chilenos nativos mantenidos en museos. Ellos lograron identificar cinco casos positivos en Rhinoderma spp., todos detectados en animales colectados entre los años 1970 y 1978, intervalo de tiempo que es coincidente con la enigmática desaparición de $R$. rufum (Soto-Azat et al. 2013b). Además, en un estudio epidemiológico transversal realizado por los mismos autores, se detectó una baja prevalencia de infección con $B d(1,9 \%)$ en $R$. darwinii, pero con altas intensidades de infección (Soto-Azat et al. 2013a). Estos autores sugieren que si $R$. darwinii fuese altamente susceptible a la quitridiomicosis, es muy probable que los individuos mueran pronto luego del comienzo de la infección, lo que llevaría a las bajas prevalencias encontradas. Respaldando esta hipótesis, los autores encontraron que: a) la prevalencia de la infección en otras especies de anuros ( $\sin$ incluir a $R$. darwinii) fue significativamente más alta en sitios con extinción o declinación poblacional de Rhinoderma spp. en comparación con sitios sin aparente declinación de Rhinoderma spp.; y b) un individuo silvestre de $R$. darwinii fue encontrado muerto y los análisis posteriores revelaron altas cargas de infección con $B d$ y hallazgos histopatológicos consistentes con quitridiomicosis (Soto-Azat et al. 2013a). A pesar de que este estudio sugiere que la quitridiomicosis podría estar asociada con la declinación y extinción de Rhinoderma spp., la evidencia de los efectos que la quitridiomicosis tiene sobre las poblaciones silvestres de Rhinoderma spp. se mantiene poco concluyente.

La búsqueda en ISI-wok no arrojó resultados de EIs afectando las poblaciones de Telmatobius spp. presentes en Chile.

Clase Aves: Azuma et al. (1988) describen la presencia del nematodo Cosmocephalus obvelatus Creplin 1825 en el esófago de dos ejemplares de Eudyptes chrysocome Forster, 1781 (E. crestatus Millar 1784) muertos en el zoológico Maruyama en Sapporo, Japón. Las aves provenían de Chile, pero no se detalla el sitio de captura (Azuma et al. 1988).

La búsqueda en ISI-wok no arrojó resultados de EIs afectando las poblaciones de E. chrysolophus.

Clase Mammalia: La lista roja menciona que Balaenoptera borealis Lesson 1828 presenta una condición patológica que deteriora las barbas de los individuos, sin embargo la etiología de esta enfermedad no es clarificada. Mizroch et al. (1984) reafirman que se trata de una enfermedad de origen desconocido, la cual perjudicaría la capacidad de los animales para alimentarse. Actualmente, la frecuencia de esta condición y su impacto en las poblaciones es desconocida (Reilly et al. 2008).

Para Lycalopex fulvipes Martin 1837 la lista roja plantea que los individuos presentes en la cordillera de Nahuelbuta podrían ser amenazados por la presencia de perros que actúen como potenciales vectores de enfermedades. GonzálezAcuña et al. (2007) describen la presencia del piojo masticador Trichodectes canis De Geer 1778 (Phthiraptera: Trichodectidae) en varios estadios (huevos, ninfas I, II, III y hembras adultas) en zorros de Chiloé, los cuales no se asociaron con lesiones en los animales infestados. Jiménez et al. (2012), en un muestreo coprológico de 189 heces de distintas localidades de Chiloé encontraron que el $21,2 \%$ de estas eran positivas a 10 endoparásitos diferentes (nematodos, cestodos y protozoos). Las tasas de infección con estos parásitos se correlacionaron mejor con la abundancia de perros en los sitios que con otras variables como el número de personas por casa. Por otra parte, Cabello et al. (2013a) realizaron análisis de PCR para detectar diversos patógenos en muestras de sangre de 30 individuos de L. fulvipes provenientes de Chiloé. Los autores encontraron 17 muestras positivas para Mycoplasma spp. (prevalencia $=56,7 \%$ ) y una muestra con la presencia concomitante de Ricketssia sp. Sin embargo, ninguno de los individuos positivos a estos patógenos presentó signos externos de enfermedad. Finalmente, Cabello et al. (2013b) detectaron la presencia de un nuevo gammaherpesvirus en muestras sanguíneas de cuatro L. fulvipes capturados en Chiloé (28 muestras fueron analizadas). Los autores destacan que la patogenicidad de este nuevo gammaherpesvirus se mantiene desconocida (Cabello et al. 2013b).

Para Hippocamelus bisulcus Molina 1782 la información de la lista roja plantea que diversas enfermedades podrían ser una mayor amenaza para su conservación, dentro de las cuales se detallan brucelosis, fiebre aftosa y cisticercosis, las cuales podrían ser transmitidas por la introducción en los rangos de hogar del huemul de especies domésticas y asilvestradas de herbívoros exóticos. Sin embargo, la evidencia de EIs presentes en la especie es escasa. González-Acuña et al. (2009) describen la presencia de huemules silvestres, capturados en la Reserva Nacional Lago Cochrane, infestados con el piojo masticador Bovicola caprae Packard 1870 (Ischnocera: Bovicoliidae) y el piojo chupador Solenopotes binipilosus Farenholz 1919 (Anoplura: Linognathidae). Además, cuatro animales presentaban la infestación con el ácaro Psoroptes ovis Hering 1838 (Acari: Psoroptidae), dos de los cuales poseían altas infestaciones con daño a la piel caracterizado por enrojecimiento, engrosamiento y 
descamación. Los autores concluyen que la significancia de esta infestación en la conservación del huemul no es clara y requiere estudios adicionales. Adicionalmente, Corti et al. (2013) detectaron que dos individuos de H. bisulcus (de 18 animales muestreados) poseían anticuerpos contra el virus de la diarrea viral bovina (BVDV, por su sigla en inglés). Los autores proponen que es muy probable que los individuos de $H$. bisulcus hayan estado expuestos al BVDV mediante el contacto con el ganado bovino y concluyen que las consecuencias de esta enfermedad en las poblaciones de H. bisulcus son desconocidas.
Nivel DE RIESGO DE AMENAZA POR EIs

De las 105 especies animales amenazadas de Chile, 13 $(12,4 \%)$ presentan riesgo muy alto, $13(12,4 \%)$ riesgo alto, 41 (39\%) riesgo medio, 21 (20\%) riesgo bajo y 17 $(16,2 \%)$ riesgo muy bajo de ser impactadas por una EI con el potencial de provocar declinaciones poblacionales o extinción. En la Tabla 2 y 3 se muestran las especies que presentan nivel de riesgo muy alto y alto, respectivamente. En el Anexo 1 se muestran las especies que presentan un nivel de riesgo medio, bajo y muy bajo.

TABLA 2. Especies animales amenazadas presentes en Chile que poseen un nivel de riesgo muy alto de ser impactadas por una enfermedad infecciosa con el potencial de producir declinaciones poblacionales o extinción.

TABLE 2. Threatened wildlife of Chile classified with very high risk of being impacted by an infectious disease with the potential to lead population decline or extinction.

\begin{tabular}{|c|c|c|c|c|c|c|}
\hline Clase & ORDEN & FAMILIA & EsPECIE & $\begin{array}{c}\text { Estado dE } \\
\text { CONSERVACIÓN }\end{array}$ & DISTRIBUCIÓN & REFERENCIAS \\
\hline \multirow{4}{*}{$\frac{. \frac{\pi}{0}}{\frac{0}{3}} \frac{1}{\frac{1}{2}}$} & \multirow{4}{*}{ Anura } & \multirow{4}{*}{ Ceratophryidae } & Telmatobius pefauri & $\begin{array}{c}\text { En peligro } \\
\text { crítico }\end{array}$ & Chile & 3,5 \\
\hline & & & $\begin{array}{c}\text { Telmatobius } \\
\text { zapahuirensis }\end{array}$ & $\begin{array}{l}\text { En peligro } \\
\text { crítico }\end{array}$ & Chile & 3,5 \\
\hline & & & $\begin{array}{l}\text { Telmatobius } \\
\text { marmoratus }\end{array}$ & Vulnerable & $\begin{array}{c}\text { Bolivia, Chile, } \\
\text { Perú }\end{array}$ & 3,5 \\
\hline & & & $\begin{array}{l}\text { Telmatobius } \\
\text { peruvianus }\end{array}$ & Vulnerable & Chile y Perú & 3,5 \\
\hline \multirow{5}{*}{ 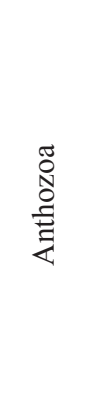 } & \multirow{5}{*}{ Scleractinia } & \multirow{5}{*}{ Acroporidae } & Acropora listeri & Vulnerable & Global & $4,6,7,9$ \\
\hline & & & $\begin{array}{c}\text { Montipora } \\
\text { australiensis }\end{array}$ & Vulnerable & Hemisferio sur & 7,10 \\
\hline & & & $\begin{array}{c}\text { Montipora } \\
\text { caliculata }\end{array}$ & Vulnerable & Hemisferio sur & 7,10 \\
\hline & & & $\begin{array}{c}\text { Montipora } \\
\text { crassituberculata }\end{array}$ & Vulnerable & Hemisferio sur & 7,10 \\
\hline & & & Pocillopora danae & Vulnerable & Hemisferio sur & $1,4,8$ \\
\hline \multirow{4}{*}{$\sum^{\infty}$} & \multirow{4}{*}{ Procellariformes } & \multirow{4}{*}{ Diomedeidae } & Diomedea sanfordi & En peligro & Hemisferio sur & 2 \\
\hline & & & $\begin{array}{c}\text { Diomedea } \\
\text { antipodensis }\end{array}$ & Vulnerable & Hemisferio sur & 2 \\
\hline & & & $\begin{array}{c}\text { Diomedea } \\
\text { epomophora }\end{array}$ & Vulnerable & Hemisferio sur & 2 \\
\hline & & & Diomedea exulans & Vulnerable & Hemisferio sur & 2 \\
\hline
\end{tabular}

\footnotetext{
${ }^{1}$ Ben-Haim et al. 2003, ${ }^{2}$ Weimerskirch 2004, ${ }^{3}$ Merino-Viteri et al. 2005, ${ }^{4}$ Dalton \& Smith 2006, ${ }^{5}$ Seimon et al. 2007, ${ }^{6}$ Miller et al. 2009, ${ }^{7}$ Sussman et al. 2008, ${ }^{8}$ Vidal-Dupiol et al. 2011, ${ }^{9}$ Gignoux-Wolfsohn et al. 2012, ${ }^{10}$ Work et al. 2012
} 
TABLA 3. Especies animales amenazadas presentes en Chile que poseen un nivel de riesgo alto de ser impactadas por una enfermedad infecciosa con el potencial de producir declinaciones poblacionales o extinción.

TABLE 3. Threatened wildlife of Chile classified with high risk of being impacted by an infectious disease with the potential to lead population decline or extinction.

\begin{tabular}{|c|c|c|c|c|c|c|}
\hline Clase & Orden & FAMILIA & ESPECIE & $\begin{array}{c}\text { ESTADO DE } \\
\text { CONSERVACIÓN }\end{array}$ & DistribuCión & REFERENCIAS \\
\hline$\frac{\pi}{\frac{\pi}{0}}$ & Anura & Bufonidae & $\begin{array}{l}\text { Rhinella } \\
\text { rubropunctata }\end{array}$ & Vulnerable & $\begin{array}{l}\text { Argentina y } \\
\text { Chile }\end{array}$ & $5,7,8$ \\
\hline \multirow{5}{*}{ 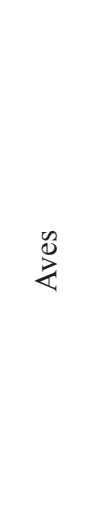 } & \multirow{5}{*}{ Procellariformes } & \multirow{5}{*}{ Diomedeidae } & $\begin{array}{c}\text { Phoebastria } \\
\text { irorrata }\end{array}$ & $\begin{array}{l}\text { En peligro } \\
\text { crítico }\end{array}$ & Océano Pacífico & 6 \\
\hline & & & $\begin{array}{l}\text { Thalassarche } \\
\text { melanophrys }\end{array}$ & En peligro & Hemisferio sur & 6 \\
\hline & & & $\begin{array}{l}\text { Thalassarche } \\
\text { chrysostoma }\end{array}$ & Vulnerable & Hemisferio sur & 6 \\
\hline & & & $\begin{array}{c}\text { Thalassarche } \\
\text { eremita }\end{array}$ & Vulnerable & Océano Pacífico & 6 \\
\hline & & & $\begin{array}{c}\text { Thalassarche } \\
\text { salvini }\end{array}$ & Vulnerable & Hemisferio sur & 6 \\
\hline \multirow{6}{*}{ 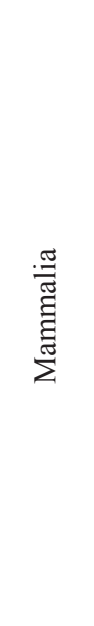 } & \multirow{3}{*}{ Carnivora } & Canidae & Lycalopex fulvipes & $\begin{array}{l}\text { En peligro } \\
\text { crítico }\end{array}$ & Chile & 1,2 \\
\hline & & \multirow{2}{*}{ Felidae } & $\begin{array}{l}\text { Leopardus } \\
\text { jacobita }\end{array}$ & En peligro & $\begin{array}{c}\text { Argentina, } \\
\text { Bolivia, Chile y } \\
\text { Perú }\end{array}$ & 3 \\
\hline & & & Leopardus guigna & Vulnerable & $\begin{array}{l}\text { Argentina y } \\
\text { Chile }\end{array}$ & 3 \\
\hline & \multirow{3}{*}{ Cetartiodactyla } & \multirow{3}{*}{ Cervidae } & $\begin{array}{l}\text { Hippocamelus } \\
\text { antisensis }\end{array}$ & Vulnerable & $\begin{array}{c}\text { Argentina, Chile } \\
\text { y Perú }\end{array}$ & 4,9 \\
\hline & & & $\begin{array}{l}\text { Hippocamelus } \\
\text { bisulcus }\end{array}$ & En peligro & $\begin{array}{l}\text { Argentina y } \\
\text { Chile }\end{array}$ & 4,9 \\
\hline & & & Pudu puda & Vulnerable & $\begin{array}{c}\text { Argentina y } \\
\text { Chile }\end{array}$ & 4,9 \\
\hline$\frac{\pi}{\stackrel{\underline{Z}}{0}}$ & Testudines & Cheloniidae & $\begin{array}{c}\text { Lepidochelys } \\
\text { olivacea }\end{array}$ & Vulnerable & Mundial & 10 \\
\hline
\end{tabular}

${ }^{1}$ Goltsman et al. 1996, ${ }^{2}$ Laurenson et al. 1998, ${ }^{3}$ Cleaveland et al. 2000, ${ }^{4}$ Gutuierrez-Espeleta et al. 2001, ${ }^{5}$ Ron et al. $2003,{ }^{6}$ Weimerskirch 2004, ${ }^{7}$ La Marca et al. 2005, ${ }^{8}$ Lampo et al. 2007, ${ }^{9}$ Tomassini et al. 2009, ${ }^{10}$ Sarmiento-Ramírez et al. 2010 


\section{DISCUSIÓN}

A pesar de que el 16,2\% de las especies animales amenazadas de Chile presentan como principal amenaza las EIs según la Lista Roja de la UICN, la búsqueda resultante del ISI-wok no arrojó evidencia de ninguna EI causando declinaciones poblacionales dentro del territorio chileno. Pese a que el ISI-wok no incluye la literatura gris, nosotros creemos que la base de datos resultante de nuestra búsqueda es representativa de la escasa evidencia existente actualmente sobre el rol de las EIs en la declinación de fauna silvestre en Chile.

Aunque el porcentaje de especies amenazadas por EIs según la lista roja es bajo, es esperable que este aumente en el futuro, especialmente debido a los cambios antropogénicos en los ecosistemas (Jones et al. 2008, Daszak et al. 2001). Por ejemplo, la introducción de especies alóctonas que porten un patógeno desconocido para especies nativas puede significar la aparición de enfermedades emergentes (Medina-Vogel 2010, Deem et al. 2001). En Argentina, Arbetman et al. (2012) detectaron la presencia del protozoo Apicystis bombi Liu, Macfarlane \& Pengelly 1974 en los abejorros exóticos Bombus terrestres Linnaeus 1758 y B. ruderatus Fabricius 1775, previamente introducidos a Chile como polinizadores (Montalva 2011, Morales 2007). Los autores plantean que la introducción de este patógeno puede estar asociada con las declinaciones poblacionales del abejorro nativo $B$. dahlbomii Guérin-Méneville 1835, el cual también está declinando en Chile (Montalva 2012). Recientemente, en el sur de Chile se detectaron ejemplares de visón americano (Neovison vison Schreber 1777) seropositivos al virus del distemper canino (CDV, por sus siglas en inglés). El hecho de que el CDV esté circulando en el ecosistema, tanto en especies domésticas como silvestres, representa un alto riesgo para especies de carnívoros chilenos amenazados tales como la nutria de río (Lontra provocax Thomas 1908) y el zorro de Darwin (L. fulvipes; Sepúlveda et al. 2014). Por otra parte, la introducción de patógenos en nuevas áreas geográficas puede ocurrir como consecuencia de planes de manejo que incluyan la translocación de animales (Deem et al. 2001), situación que representa una seria amenaza para los programas de conservación (Cunningham 1996). Otros cambios ambientales, tales como la fragmentación y pérdida del hábitat, la contaminación y el cambio climático pueden afectar la dinámica de las EIs, produciendo la emergencia de nuevas enfermedades que aumenten el riesgo de extinción de animales silvestres (Medina-Vogel 2010, Smith et al. 2009, Collinge et al. 2005, Harvell et al. 2002, Githeko et al. 2000).

Los biólogos de la conservación están comenzando a entender el rol de los patógenos en la poblaciones naturales; sin embargo, es imperativo focalizar los esfuerzos en las especies amenazadas y sus enfermedades, de tal forma que sea posible generar estrategias de control y manejo (Smith et al. 2009). En este sentido, en el presente trabajo nosotros asignamos a cada especie animal amenazada de Chile un nivel de riesgo de ser impactada por una EI que tenga el potencial de provocar declinaciones poblacionales o extinción. Esta categorización se basó en la relación de cercanía taxonómica que tenía cada especie amenazada con otra especie para la cual la literatura indexada en ISI-wok describe mortalidad en masa, declinación poblacional o extinción mediada por patógenos. Esta categorización, pese a que posiblemente se encuentra sesgada por un diferencial en el esfuerzo de investigación entre grupos taxonómicos, la proponemos como una herramienta que puede ser utilizada para priorizar el monitoreo epidemiológico y para desarrollar futuras estrategias de prevención y control de EIs de la fauna silvestre del país.

Especial atención deberían recibir especies endémicas de distribución restringida y que se encuentran en peligro crítico de extinción, debido a que la evidencia indica que el factor mayormente asociado a extinción inducida por enfermedad es un pequeño tamaño poblacional previo a la epidemia (de Castro \& Bolker 2005). Entre las especies que poseen ambas características y que además presentan un riesgo alto y muy alto de ser impactadas por una EI, se encuentran los anuros Telmatobius zapahuirensis Veloso, Sallaberry, Navarro, Iturra-C, Valencia, Penna \& Díaz 1982 y T. pefauri Veloso \& Trueb 1976, y el cánido L. fulvipes. Cabe destacar que para T. marmoratus Duméril \& Bibron 1841, especie que habita Bolivia, Chile y Perú, Seimon et al. (2007) detallan la presencia de varios individuos infectados con el hongo quítrido $(B d)$ en 2002-2003 en la Cordillera Vilcanota, Perú. Los individuos colectados presentaban signos clínicos y patológicos de quitridiomicosis fatal, incluyendo letargia a la manipulación, hiperplasia marcada de la línea queratinocítica de la piel e infección intensa (alto número de esporangios). Este hecho se correlacionó con un evento de mortalidad en masa observado el 2004 y con la aparente desaparición de pre y post-matamórficos para marzo de 2005 (Seimon et al. 2007). La alta susceptibilidad de $T$. marmoratus a la infección por $B d$ y la sospechada para otras especies del género Telmatobius (Merino-Viteri et al. 2005), sumado a la cercanía geográfica del evento de mortalidad observado en T. marmoratus en relación con la localidad de T. zapahuirensis y T. pefauri (Zapahuira, Provincia de Parinacota), destacan la urgente necesidad de realizar estudios que aborden temáticas relacionadas con el estado sanitario y poblacional de T. zapahuirensis y T. pefauri en el norte del país. Por otra parte, L. fulvipes podría verse severamente afectada por el contacto con perros domésticos que actúen como reservorios y vectores de diversas enfermedades, entre ellas el distemper canino (Jiménez et al. 2008, de Castro \& Bolker 2005). El 
"derramamiento" ("spillover" sensu Daszak et al. 2000) de patógenos desde especies domésticas hacia silvestres ha sido identificado en diversas especies a lo largo del mundo, tales como abejorros (Bombus spp., Colla et al. 2006), leones (Phantera leo, Cleaveland et al. 2000), lobos etíopes (Canis simensis Rüppell 1840; Laurenson et al. 1997, 1998) y nutrias de mar (Enhydra lutris nereis Linnaeus 1758, Kreuder et al. 2003). En este contexto, Acosta-Jamett et al. (2011) proponen que las poblaciones de perros domésticos fueron la fuente de infección del virus del distemper canino en el brote ocurrido el año 2003 en los zorros silvestres Lycalopex griseus Gray 1837 y L. culpaeus Molina 1782 en la región de Coquimbo, Chile (ver también Rubio et al. 2013). Los antecedentes recopilados por los autores ponen sobre manifiesto la necesidad de dedicar esfuerzos de investigación a la interacción epidemiológica entre perro y L. fulvipes, con la finalidad de evaluar el impacto real del perro doméstico en la transmisión de enfermedades y la declinación de este zorro endémico y en peligro crítico de extinción.

\section{DiRECCIONES FUTURAS}

Aunque en un bajo porcentaje de las especies de fauna silvestre amenazada de Chile se menciona como principal amenaza una EI, esto puede ser una subestimación de la realidad como resultado de una inadecuada capacidad en el diagnóstico y monitoreo de los impactos que tienen las EIs en las poblaciones silvestres en Chile. Por esto, resulta necesario: i) generar información de base para conocer la real situación de las EIs en la fauna silvestre, especialmente en especies amenazadas con un alto riesgo de ser impactadas por EIs, ii) investigar la estructura inmunogenética de poblaciones amenazadas, además de los cambios ecoinmunológicos que se puedan generar producto del cambio climático proyectado, y así identificar poblaciones que se encuentren en mayor riesgo de extinción mediada por EIs, iii) desarrollar planes de vigilancia epidemiológica que nos permitan identificar cambios en la ecología de las enfermedades infecciosas, con el fin de detectar la aparición de nuevos brotes que amenacen la salud de los animales silvestres, domésticos y del ser humano. Finalmente, iv) promover la medicina de la conservación; transdisciplina que conjuga la salud de la fauna silvestre, del ser humano y los cambios ambientales, con el fin de contribuir a alcanzar la meta de la conservación biológica en Chile.

\section{AGRADECIMIENTOS}

Agradecemos a Federico Villatoro, Cayetano Espinosa, Emma Stapleton, Marcelo Fuentes-Hurtado y a dos revisores anónimos por sus importantes comentarios que contribuyeron a mejorar el manuscrito. Este estudio fue llevado a cabo como parte del cumplimiento del grado de
Doctor en Medicina de la Conservación (por A.V-S) de la Facultad de Ecología y Recursos Naturales, Universidad Andres Bello, Chile.

\section{BIBLIOGRAFÍA}

Acosta-Jamett, G., Chalmers, W.S.K., Cunningham, A.A., Cleaveland, S., Handel, I.G. \& Bronsvoort, B.M. 2011. Urban domestic dog populations as a source of canine distemper virus for wild carnivores in the Coquimbo region of Chile. Veterinary Microbiology 152:247-257.

Arbetman, M.P., Meeus, I., Morales, C.I., Aizen, M.A. \& Smagghe, G. 2012. Alien parasite hitchhikes to Patagonia on invasive bumblebee. Biological invasions DOI 10.1007/s10530012-0311-0.

Azuma, H., Oкамoto, M., Ohbayashi, M., Nishine, Y. \& Mukai, T. 1988. Cosmocephalus obvelatus (Creplin 1825) (Nematoda: Acuariidae) collected from the esophagus of rockhopper penguin, Eudyptes crestatus. Japanesse Journal Veterinary Research 36:73-77.

Ben-Haim, Y., Zicherman-Keren, M. \& Rosenberg, E. 2003. Temperature-Regulated Bleaching and Lysis of the Coral Pocillopora damicornis by the Novel Pathogen Vibrio coralliilyticus. Applied and Environmental Microbiology 69:4236-4242.

Bourke, J., Mutschmann, F., Ohst, T., Ulmer, P., Gutsche, A., Busse, K., Werning, H. \& Boenme, W. 2010. Batrachochytrium dendrobatidis in darwin's frog Rhinoderma spp. in Chile. Diseases of Aquatic Organisms 92:217-22.

Cabello, J., Altet, L., Napolitano, C., Sastre, N., Hidalgo, E., Dávila, J.A. \& Millán, J. 2013a. Survey of infectious agents in the endangered Darwin's fox (Lycalopex fulvipes): high prevalence and diversity of hemotrophic mycoplasmas. Veterinary Microbiology 167:448-454.

Cabello, J., Esperón, F., Napolitano, C., Hidalgo, E., Dávila, J.A. \& Millán, J. 2013b. Molecular identification of a novel gammaherpesvirus in the endangered Darwin's fox (Lycalopex fulvipes). Journal of General Virology 94:2745-2749.

Cleaveland, S., Appel, M.G.J., Chalmers, W.S.K., Chillingworth, C., Kaare, M. \& Dye. C. 2000. Serological and demographic evidence for domestic dogs as a source of canine distemper virus infection for Serengeti wildlife. Veterinary Microbiology 72:217-227.

Colla, S.R., Otterstatter M.C., Gegear, R.C. \& Thomson, J.D. 2006. Plight of the bumble bee: Pathogen spillover from commercial to wild populations. Biological conservation 129:461-467.

Collinge, S.K., Johnson, W.C., Ray, C., Matchett, R., Grensten, J., Cully, J.F., Gage, K.L., Kosoy, M.Y., Loye, J.E. \& Martin, A.P. 2005. Landscape structure and plague occurrence in black-tailed prairie dogs on grasslands of the western USA. Landscape Ecology 20:941-955.

Corti, P., Saucedo, C. \& Herrera, P. 2013. Evidence of bovine viral diarrhea, but absence of infectious bovine rhinotracheitis and bovine brucellosis in the endangered huemul deer (Hippocamelus bisulcus) in Chilean Patagonia. Journal of 
Wildlife diseases 49:744-746.

Cunningham, A.A. \& Daszak, P. 1998. Extinction of a species of land snail due to infection with a microsporidian parasite. Conservation Biology 12:1139-1141.

Cunningham, A.A. 1996. Disease Risks of Wildlife Translocations. Conservation Biology 10:349-353.

Dalton, S.J. \& Smith, S.D.A. 2006. Coral disease dynamics at a subtropical location, Solitary Islands Marine Park, eastern Australia. Coral Reefs 25:37-45.

Daszak, P., Cunningham, A.A. \& Hyatt A.D. 2000. Emerging infectious diseases of wildlife- Threats to biodiversity and human health. Science 287:443-449.

Daszak, P., Cunningham, A.A. \& Hyatt, H.D. 2001. Anthropogenic environmental change and the emergence of infectious diseases in wildlife. Acta Tropica 78:103-116.

Daszak, P., Cunningham, A.A. \& Hyatt, H.D. 2003. Infectious disease and amphibian population declines. Diversity and Distribution 9:141-150.

De Castro, F. \& Bolker, B. 2005. Mechanisms of disease-induced extinction. Ecology Letters 8:117-126.

Deem, S.L., Karesh, W.B. \& Weisman, W. 2001. Putting theory into practice: wildlife health in conservation. Conservation Biology 15:1224-1233.

Gaydos, J.K. \& Gilardi, K.V. 2004. Addressing disease risks when recovering species at risk. En: Proceedings of the Species at Risk 2004 Pathways to Recovery Conference. (Ed. Hooper., T.). Victoria, Columbia Británica, Canadá.

Gignoux-Wolfsohn, S.A., Marks, C.J. \& Vollmer, S.V. 2012. White Band Disease transmission in the threatened coral, Acropora cervicornis. Scientific Reports 2:804.

Githeko, A.K., Lindsay, S.W., Confalonieri, U.E. \& Patz, J.A. 2000. Climate change and vector-borne diseases: a regional analysis. Bulletin of the World Health Organization 78:1136-1147.

Goltsmam, M., Kruchenkova, E.P. \& Macdonald, D.W. 1996. The Mednyi Arctic foxes: treating a population imperilled by disease. Oryx 30:251-258.

González-Acuña, D., Briceño, C., Cicchino, A., Funk, S.M. \& JimÉneZ, J. 2007. First records of Tricodectes canis (Insecta: Phthiraptera: Trichodectidae) from Darwin's fox, Pseudalopex fulvipes (Mammalia: Carnivora: Canidae). European Journal of Wildlife Research 53:76-79.

González-Acuña, D., Saucedo, C., Corti, P., Casanueva, M.E. \& Cicchino, A. 2009. First records of the louse Solenopotes binipilosus (Insecta: Phthiraptera) and mite Psoroptes ovis (Arachnida: Acari) from wild southern huemul (Hippocamelus bisulcus). Journal of Wildlife Diseases 45:1235-1238.

Gutierrez-Espeleta, G.A., Hedrick, P.W., Kalinowski, S.T., Garrigan, D. \& Boyce, W.M. 2001. Is the decline of desert bighorn sheep from infectious disease the result of low MHC variation? Heredity 86:439-450.

Harvell, C.D., Mitchell, C.E., Ward, J.R., Altizer, S., Dobson, A.P., Ostreld, R.S. \& Samuel, M.D. 2002. Climate warming and disease risks for terrestrial and marine biota. Science 296:2158-2162.

Hinojosa-SÁez, A. \& GonzÁlez-Acuña, D. 2005. Estado actual del conocimiento de helmintos en aves silvestres de Chile. Gayana 69:241-253.

Jiménez, J.E., Briceño, C., Alcaíno, H., VÁsquez, P., Funk, S.
\& GonZÁlez-AcuñA, D. 2012. Coprologic survey of endoparasites from Darwin's fox (Pseudalopex fulvipes) in Chiloé, Chile. Archivos de Medicina Veterinaria 44:93-97.

Jiménez, J.E., Lucherini, M. \& Novaro, A.J. 2008. Pseudalopex fulvipes. IUCN Red List of Threatened Species. Version 2012.2. URL: www.iucnredlist.org. Accesado: 25 marzo, 2013.

Jones, K.E., Patel, N.G., Levy, M.A., Storeygard, A., Balk, D., Gittleman, J.L. \& DaszaK, P. 2008. Global trends in emerging infectious diseases. Nature 451:990-994.

Kreuder, C., Miller, M.A., Jessup, D.A., Lowenstine, L.J., Harris, M.D., Ames, J.A., Carpenter, T.E., Conrad, P.A. \& MAZET, J.A. 2003. Patterns of mortality in southern sea otters (Enhydra lutris nereis) from 1998-2001. Journal of Wildlife Diseases 39:495-509.

La Marca, E., Lips, K.R., Otters, S.L., Puschendorf, R., Ibáñez, R., Rueda-Almonacid, JV., Schulte, R., Marty, C., Castro, F., Manzanilla-Puppo, J., García-Pérez, J.E., Bolaños, F., Chaves, G., Pounds, J.A., Toral, E. \& Young, B.E. 2005. Catastrophic Population declines and extinctions in neotropical Harlequin Frogs (Bufonidae: Atelopus). Biotropica 37:190-201.

LAFFERTY, K.D. 2003. Is disease increasing or decreasing, and does it impact or maintain biodiversity? Journal of Parasitology 89:s101-s105.

LAMPO, M., BARrIO-AMORÓs, C. \& HAN, B. 2007. Batrachochytrium dendrobatidis infection in the recently rediscovered Atelopus mucubajiensis (Anura, Bufonidae), a critically endangered Frog from the Venezuelan Andes. EcoHealth 3:299-302

Laurenson, K., Shiferaw, F. \& Sillero-Zubiri, C. 1997. Disease, Domestic Dogs and the Ethiopian wolf: the Current Situation. En: The Ethiopian wolf - Status, Survey and Conservation Action Plan. (Eds. Sillero-Zubiri, C. \& D. Mcdonald), pp. 32-42. IUCN, Gland, Suiza.

Laurenson, K., Sillero-Zubiri, C., Thompson, H., Shiferaw, F., Thirgood, S., Malcolm, J. 1998. Disease as a threat to endangered species: Ethiopian wolves, domestic dogs and canine pathogens. Animal Conservation 1:273-280.

Leendertz, F.H., Pauli, G., Ellerbrok, H., Maetz- Rensing, K., BoARDMAN, W., NunN,

C., Ellerbrok, H., Jensen, S.A., Junglen, S. \& Boesch, C. 2006. Pathogens as drivers of population declines: The importance of systematic monitoring in great apes and other threatened mammals. Biological Conservation 131:323-337.

Leopold, A. 1933. Game management. Scribner's, New York. 481 pp.

MAY, R.M. 2010. Ecological science and tomorrow's world. Philosophical Transactions of the Royal Society B 365:4147.

Medina-Vogel, G. 2010. Ecología de enfermedades infecciosas emergentes y conservación de especies silvestres. Archivos de Medicina Veterinaria 42:11-24.

Merino-Viteri, A., Coloma, L.A. \& Almendáriz, A. 2005. Los Telmatobius de los Andes de Ecuador y su disminución poblacional. Monografías de Herpetología 7:9-37.

Millennium Ecosystem Assessment. 2005. Ecosystems and human well-being: synthesis. Island Press, Washington DC.

Miller, J., Muller, E., Rogers, C., Waara, R., Atkinson, A., 
Whelan, K.R.T., Patterson, M. \& Witcher, B. 2009. Coral disease following massive bleaching in 2005 causes $60 \%$ decline in coral cover on reefs in the US Virgin Islands. Coral Reefs 28:925-937.

Mizroch, S.A, Rice, D.W. \& Breiwick, J.M. 1984. The fin whale, Balaenoptera physalus. Marine Fisheries Review 46:2024.

Montalva, J. 2012. La difícil situación del abejorro más austral del mundo (Bombus dahlbomii Guérin-Méneville, 1835). Boletín de Biodiversidad de Chile 7:1-3.

Montalva, J., Dudley, L., Arroyo, M.K., Retamales, H. \& Aвrahamovich, A.H. 2011. Geographic distribution and associated flora of native and introduced bumble bees (Bombus spp.) in Chile. Journal of Apicultural Research 50:11-21.

Morales, C.L. 2007. Introducción de abejorros (Bombus) no nativos: causas, consecuencias ecológicas y perspectivas. Ecología Austral 17:51-65.

Pedersen, A.B., Jones, K.E., Nunn, C.L. \& Altizer, S. 2007. Infectious diseases and extinction risk in wild mammals. Conservation Biology 21:1269-1279.

Reilly, S.B., Bannister, J.L., Best, P.B., Brown, M., Brownell Jr., R.L., Butterworth, D.S., Clapham, P.J., Cooke, J., Donovan, G.P., Urbán, J. \& Zerbini, A.N. 2008. Balaenoptera borealis. En: IUCN 2012. IUCN Red List of Threatened Species. Version 2012.2. <www. iucnredlist.org>. Accedido el 10 de noviembre de 2012.

Robinson, R.A., Lawson, B., Toms, M.P., Peck, K.M., Kirkwood, J.K., Chantrey, J., Clatworthy, I.R., Evans, A.D., Hughes, L.A., Hutchinson, O.C., John, S.K., Pennycott, T.W., Perkins, M.W., Rowley, P.S., Simpson, V.R., Tyler, K.M., Cunningham, A.A. 2010. Emerging infectious disease leads to rapid population declines of common british birds. PLoS ONE 5: e12215. doi:10.1371/journal. pone. 0012215 .

Rodrigues, A.S.L., Pilgrim, J.D., Lamoreux, J.F., Hoffmann, M. \& BRooks, T.M. 2006. The value of the IUCN Red List for conservation. TRENDS in Ecology and Evolution 21:7176.

Ron, S.R., Duellman, W.E., Coloma, L.A. \& Bustamante, M.R. 2003. Population Decline of the Jambato Toad Atelopus ignescens (Anura: Bufonidae) in the Andes of Ecuador. Journal of Herpetology 37:116-126.

Rubio, A.V., Fredes, F. \& Bonacic, C. 2013. Serological and parasitological survey of free-ranging culpeo foxes (Lycalopex culpaeus) in the mediterranean biodiversity hotspot of central Chile. Journal of Animal and Veterinary Advances 12:1445-1449.

Sarmiento-Ramírez, J.M., Abella, E., Martín, M.P., Tellería, M.T., López-Jurado, L.F., Marco, A. \& DiéGuez-
Uribeondo, J. 2010. Fusarium solani is responsible for mass mortalities in nests of bloggerhead sea turtle, Caretta caretta, in Boa vista, Cape Verde. FEMS Microbiology Letters 312:192-200.

Seimon, T.A., Seimon, A., Daszak, P., Halloy, S.R., Schloegel, L.M., Aguilar, C.A., Sowell, P., Hyatt, A.D., Konecky, B. \& Simmons, J.E. 2007. Upward range extension of Andean anurans and chytridiomycosis to extreme elevations in response to tropical deglaciation. Global Change Biology 12:1-12.

Sepúlveda, M.A., Singer, R.S., Silva-Rodríguez, E.A., Eguren, A., Stowhas, P. \& Pelican, K. 2014. Invasive American mink: linking pathogen risk between domestic and endangered carnivores. EcoHealth. DOI: 10.1007/s10393-014-0917-z

Smith, K.F., SAX, D.F. \& LAFFerty, K.D. 2006. Evidence for the Role of Infectious Disease in Species Extinction and Endangerment. Conservation Biology 20:1349-1357.

Smith, K.F., Acevedo-Whitehouse, K. \& Pedersen, A.B. 2009. The role of infectious diseases in biological conservation. Animal Conservation 12:1-12.

Soto-Azat, C., Valenzuela-Sánchez, A., Clarke, B.T., Busse, K., Ortiz, J.C., Barrientos, C. \& Cunningham, A.A. 2013 a. Is chytridiomycosis driving Darwin's frogs to extinction? PLoS one 8:e79862.

Soto-Azat, C., Valenzuela-Sánchez, A., Collen, B., Rowcliffe, J.M., Veloso, A. \& Cunningham, A.A. 2013b. The population decline and extinction of Darwin's frogs. PLoS one 8: e66957.

Sussman, M., Willis, B.L., Victor, S. \& Bourne, D.G. 2008. Coral Pathogens Identified for White Syndrome (WS) Epizootics in the Indo-Pacific. PLoS one 3:e2393.

Tomassini, L., Gonzales, B., Weiser, G.C. \& Sischo, W. 2009. An ecologic study comparing distribution of Pasteurella trehalosi and Mannheimia haemolytica between Sierra Nevada bighorn sheep, White Mountain bighorn sheep, and domestic sheep. Journal of Wildlife Diseases 45:930940.

Vidal-Dupiol, J., Ladrière, O., Meistertzheim, A., Fouré, L., Adjeroud, M. \& Mitta, M. 2011. Physiological responses of the scleractinian coral Pocillopora damicornis to bacterial stress from Vibrio coralliilyticus. The Journal of Experimental Biology 214:1533-1545.

WeIMERSKIRCH, H. 2004. Diseases threaten Southern Ocean albatrosses. Polar Biology 27:374-379.

WERNING, H. 2009. From darwin's treasure chest: Rhinoderma. IRCF Reptiles and Amphibians 16:247-255.

Work, T.M., Russell, R. \& Aeby, G.S. 2012. Tissue loss (white syndrome) in the coral Montipora capitata is a dynamic disease with multiple host responses and potential causes. Proceedings of the Royal Society B 279:4334-4341. 
APÉnDiCe 1. Especies animales amenazadas de Chile (Lista Roja de la UICN) que presentan nivel de riesgo medio, bajo y muy bajo de ser impactadas por una enfermedad infecciosa con el potencial de producir declinaciones poblacionales o extinción.

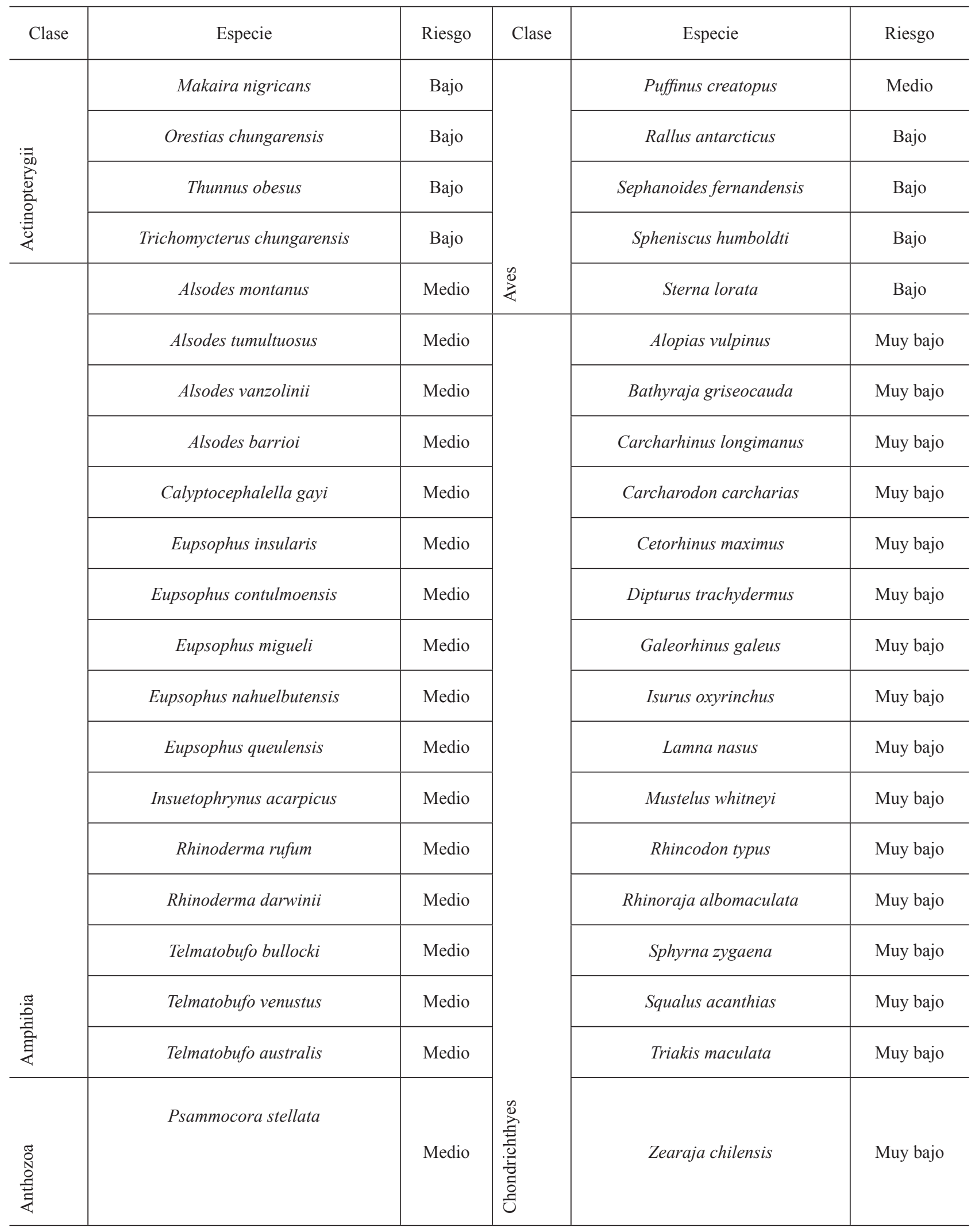


Enfermedades infecciosas de la fauna silvestre: AndRÉs VAlEnZuEla-SÁnchEZ \& GonZALo Medina-Vogel

\begin{tabular}{|c|c|c|c|c|c|}
\hline & Agriornis albicauda & Medio & 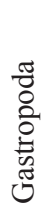 & Chilina angusta & Muy bajo \\
\hline & Aphrastura masafuerae & Medio & \multirow{3}{*}{ 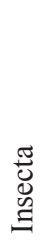 } & Antiagrion blanchardi & Bajo \\
\hline & Conirostrum tamarugense & Medio & & Phyllopetalia altarensis & Bajo \\
\hline & Eudyptes chrysocome & Bajo & & Phyllopetalia excrescens & Bajo \\
\hline & Eudyptes chysolophus & Bajo & & Amorphochilus schnablii & Medio \\
\hline & Eulidia yarrellii & Bajo & & Balaenoptera borealis & Medio \\
\hline & Nesofregetta fuliginosa & Medio & & Balaenoptera musculus & Medio \\
\hline & Numenius Borealis & Bajo & & Balaenoptera physalus & Medio \\
\hline & Numenius tahitiensis & Bajo & & Chaetophractus nationi & Medio \\
\hline & Pelecanoides garnotti & Medio & & Chinchilla chinchilla & Bajo \\
\hline & Phoenicoparrus andinus & Bajo & & Chinchilla lanigera & Bajo \\
\hline & Podiceps gallardoi & Bajo & & Ctenomys magellanicus & Medio \\
\hline & Procellaria aequinoctialis & Medio & & Lontra felina & Medio \\
\hline & Procellaria westlandica & Medio & & Lontra provocax & Medio \\
\hline & Pterodroma cookii & Medio & & Octodon pacificus & Bajo \\
\hline & Pterodroma difilippiana & Medio & & Octodon bridgesi & Medio \\
\hline & Pterodroma externa & Medio & & Pearsonomys annectens & Medio \\
\hline & Pterodroma longirostris & Medio &.$\stackrel{\pi}{]}$ & Physeter macrocephalus & Medio \\
\hline 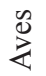 & Puffinus bulleri & Medio & $\stackrel{\bar{\Xi}}{\Sigma}^{\bar{E}}$ & & \\
\hline
\end{tabular}

Recibido: 30.03 .13

Aceptado: 09.05.14 